\title{
Levels of bone marrow microvessel density are crucial for evaluating the status of acute myeloid leukemia
}

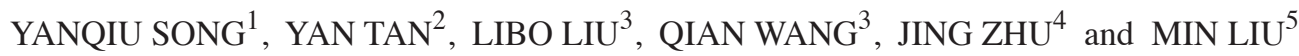 \\ ${ }^{1}$ Cancer Center, The First Hospital, Jilin University; ${ }^{2}$ Department of Oncology, Jilin Province People's Hospital; \\ ${ }^{3}$ Department of Hematology, The Fourth Hospital, Jilin University; ${ }^{4}$ Department of Oncology, \\ Jilin Cancer Hospital; ${ }^{5}$ Department of Radiation Oncology, The First Hospital, \\ Jilin University, Changchun, Jilin 130021, P.R. China
}

Received August 1, 2014; Accepted April 14, 2015

DOI: $10.3892 / 01.2015 .3209$

\begin{abstract}
The aim of the present study was to determine the efficacy of bone marrow microvessel density (BM-MVD) in the evaluation of the status of acute myeloid leukemia (AML). The levels of serum and bone marrow vascular endothelial growth factor (VEGF), and BM-MVD in 28 patients with AML, 10 patients with non-Hodgkin's lymphoma, 10 patients with anemia and 14 patients with AML that achieved complete remission for six months (AML-DFS) subsequent to chemotherapy were determined by ELISA and immunohistochemistry. The levels of serum VEGF in patients with hematological disorders were significantly increased compared with the levels in the healthy controls. The levels of BM VEGF and BM-MVD in AML patients were significantly higher compared with the levels in the patients with non-Hodgkin's lymphoma or anemia. Following chemotherapy, the levels of serum VEGF significantly increased and the levels of BM VEGF decreased in the AML patients, regardless of their therapeutic responses, compared with the levels prior to treatment. By contrast, the levels of BM-MVD in the AML patients were significantly reduced in the patients that completely recovered from AML (AML-DFS group), compared with those in other groups. The present data indicate that the levels of BM-MVD are valuable for evaluating the status of AML.
\end{abstract}

\section{Introduction}

Leukemia is a hematological neoplasm that is characterized by the uncontrolled proliferation of immature leukocytes (1-3).

Correspondence to: $\mathrm{Dr}$ Min Liu, Department of Radiation Oncology, The First Hospital, Jilin University, 71 Xinmin Street, Changchun, Jilin 130021, P.R. China

E-mail: liumin602822@163.com

Key words: vascular endothelial growth factor, bone marrow microvessel density, acute myeloid leukemia
Currently, the incidence of leukemia is increasing worldwide (4). In 2012, a total of 47,150 new cases were diagnosed in the United States $(5,6)$. Although leukemia has been considered to be a treatable disease, the rates of successful treatment and long-term survival rate remain low, particularly for patients with certain types of leukemia, such as those with complex karyotypes (7). Previous studies have demonstrated that genetic mutations and environmental factors are associated with the development of leukemia (8-10). However, the pathogenesis of leukemia and factors that regulate leukemic cell proliferation are not fully understood.

Previous studies have indicated that bone marrow angiogenesis is crucial for the pathogenesis of human leukemia (11). Vascular endothelial growth factor (VEGF) is a growth factor that stimulates vasculogenesis and angiogenesis (12). The VEGF receptors (VEGFRs) are members of the tyrosine kinase c-fms family of proteins (13). There are three VEGFRs, the fms-like tyrosine kinase-1 (Flt-1), also termed VEGFR-1, and the kinase insert domain-containing receptors (KDRs), also termed VEGFR-2 and VEGFR-3 (14). VEGF plays key roles in angiogenesis, physiological embryogenesis, pathological tumorigenesis and metastasis (15-18). While treatment with bevacizumab to neutralize VEGF has been revealed to inhibit the growth of solid tumors in human patients and rodent models of cancer, VEGF is also a crucial factor for the proliferation of immature leukocytes and leukemic cells in the bone marrow (19). Increased levels of serum VEGF and bone marrow microvessel density (BM-MVD) are observed in patients with various types of hematological malignancies and are associated with the severity of disease in patients with leukemia (20-23). Decreased levels of BM-MVD and serum VEGF are detected in patients with acute myeloid leukemia (AML) subsequent to remission (24). Furthermore, increased levels of BM-MVD are associated with a poor prognosis in patients with AML (21). However, there is little information on the presence of altered levels of BM-MVD and serum VEGF in Chinese patients with hematological neoplasms.

In the present study, the levels of BM-MVD, BM VEGF and serum VEGF were examined in 62 Chinese patients with various hematological disorders, prior to and following the administration of standard chemotherapies, and the implications of the present findings are discussed. 


\section{Materials and methods}

Subjects. A total of 28 patients with newly-diagnosed AML, 10 patients with non-Hodgkin's lymphoma (NHL) and 10 patients with anemia were recruited at the inpatient service of the Department of Hematology and Oncology of the First Hospital, Jilin University (Changchun, China), between August 2003 and October 2012. An additional 14 patients that had experienced complete remission of AML for 6 months [AML-disease-free survival (DFS)] following the administration of standard therapies were also enrolled. In total, 10 gender- and age-matched healthy control (HC) individuals were recruited and served as the control group. The characteristics of patients with various conditions are presented in Table I. Individual patients with AML were diagnosed according to the criteria of the World Health Organization (25) and classified according to the criteria of the French-American-British classification system (26), based on the results of morphological, immunohistological, cytological, molecular and biochemical examinations of the bone marrow and peripheral blood specimens. The patients with NHL were diagnosed according to immunophenotypical criteria (27) subsequent to the analysis of biopsied tumor specimens. Patients with anemia were diagnosed according to the results of laboratory examinations. Patients were excluded from the present study if dysfunction of vital organs, mental illness or another type of malignancy were present, or if the patient was pregnant. Written informed consent was obtained from individual patients, and the experimental protocol was approved by the Institutional Review Committee of Jilin University and the ethics committee of The First Hospital, Jilin University.

Sample collection. Fasting venous blood samples and samples obtained from bone marrow aspiration and biopsy were collected from individual patients, as previously described (20-24). The individual blood and bone marrow aspiration samples were subjected to centrifugation at $1,000 \mathrm{x}$ g for $10 \mathrm{~min}$, and the resulting plasma samples and bone marrow supernatants were stored at $-80^{\circ} \mathrm{C}$.

Treatment. Patients with acute promyelocytic leukemia (M3 AML) were treated with a daily dose of 8-10 mg arsenic trioxide for 28 days, and patients with other types of AML (M0, M1, M2, M4, M5, M6 and M7) were treated with the HA protocol, consisting of a homoharringtonine base with cytarabine, DA protocol, consisting of daunorubicin and cytarabine, or IDA protocol, consisting of idarubicin and cytarabine. Patients with NHL were treated using the CHOP protocol, consisting of cyclophosphamide, doxorubicin, vincristine and prednisone, or the R-CHOP protocol, consisting of rituximab, cyclophosphamide, doxorubicin, vincristine and prednisone. Patients with anemia were treated with a supplement of hematogenic materials. A total of 16 patients with AML underwent blood and bone marrow sampling again at 28-30 days subsequent to the previous treatment. The therapeutic efficacy of individual patients was evaluated, according to the previously reported criteria (28).

Immunohistochemistry. The BM-MVD of individual biopsy samples was determined by immunohistochemistry, as previously described $(26,28)$. Briefly, the bone marrow biopsy
Table I. Patient characteristics.

\begin{tabular}{|c|c|}
\hline Characteristic & Value, n (\%) \\
\hline \multicolumn{2}{|l|}{ Age } \\
\hline Range, years & $16-66$ \\
\hline$\leq 40$ years & $28(45)$ \\
\hline$>40$ years & $34(55)$ \\
\hline \multicolumn{2}{|l|}{ Gender } \\
\hline Male & $27(44)$ \\
\hline Female & $35(56)$ \\
\hline \multicolumn{2}{|l|}{$\begin{array}{l}\text { AML (firstly diagnosed } \\
\text { without treatment) }\end{array}$} \\
\hline M1 (HA/DA/IDA) & $2(3)$ \\
\hline M2 (HA/DA/IDA) & $5(8)$ \\
\hline M4 (HA/DA/IDA) & $5(8)$ \\
\hline M5 (HA/DA/IDA) & $3(4)$ \\
\hline M6 (HA/DA/IDA) & $1(2)$ \\
\hline M7 (HA/DA/IDA) & $1(2)$ \\
\hline M3 (As2O3) & $11(18)$ \\
\hline \multicolumn{2}{|l|}{ Disease type } \\
\hline $\begin{array}{l}\text { NHL without } \\
\text { (CHOP/R-CHOP) bone } \\
\text { marrow involvement }\end{array}$ & $10(16)$ \\
\hline $\mathrm{IDA}(\mathrm{Fe})$ & $6(10)$ \\
\hline MA (folic acid VitB12) & $4(6)$ \\
\hline AML-DFS & $14(23)$ \\
\hline
\end{tabular}

AML, acute myeloid leukemia; AML-DFS, disease free survival of AML 6 months subsequent to chemotherapy; HA, homoharringtonine base with cytarabine; DA, daunorubicin and cytarabine; IDA, idarubicin and cytarabine; CHOP, cyclophosphamide, doxorubicin, vincristine and prednisone; R-CHOP, rituximab, cyclophosphamide, doxorubicin, vincristine and prednisone.

samples were fixed in cold acetone for $2 \mathrm{~h}$ and embedded in alcohol-ethylene methacrylate (The Sixth People's Hospital of Shanghai, Shanghai, China). The bone marrow tissue samples were sliced into $4-\mu \mathrm{M}$ thick sections, and were treated with $0.4 \%$ pepsin and then $3 \%$ methanol, followed by blocking with $10 \%$ goat sera. Subsequently, the tissue sections were stained with polyclonal rabbit anti-human von Willebrand factor antibodies (vWF; dilution, 1:500; RAB-0068; Maixin Biotechnology, Fuzhou, Fujian, China) overnight at $4^{\circ} \mathrm{C}$, as $\mathrm{vWF}$ is expressed by megakaryocytes and endothelial cells and easily identified microvascular endothelial cells (26). By contrast, other biomarkers, such as cluster of differentiation (CD)31, CD34 or Ulex europaeus agglutinin 1, are not easily used for the measurement of BM-VMD. Subsequent to being washed, the bound antibodies were detected using horseradish peroxidase (HRP)-conjugated goat anti-rabbit IgG and visualized using diaminobenzidine (DAB). The control sections were treated with rabbit sera from healthy animals. The numbers of microvessels were determined in a blinded manner, as previously described $(24,29,30)$. First, three hot-spot areas of anti-vWF staining were identified (magnification, x100) and 
Table II. Levels of serum VEGF, BM-VEGF and BM-MVD in various groups of patients.

\begin{tabular}{lcccc}
\hline & HC & AML & NHL & Benign anemia \\
\hline B-VEGF, pg/ml & $41.76 \pm 10.03$ & $74.97 \pm 29.04$ & $146.89 \pm 74.67$ & $122.05 \pm 45.23$ \\
BM-VEGF, pg/ml & & $150.58 \pm 73.36$ & $100.39 \pm 28.10$ & $94.85 \pm 30.55$ \\
MVD & & $22.28 \pm 6.89$ & $7.76 \pm 2.24$ & $11.11 \pm 2.35$ \\
\hline
\end{tabular}

The MVD value is presented as the number of microvessels per high-power field (magnification, x400). VEGF, vascular endothelial growth factor; B-VEGF, blood plasma VEGF; BM-VEGF, bone marrow VEGF; MVD, microvessel density; AML, acute myeloid leukemia; HC, healthy controls; NHL, non-Hodgkin's lymphoma.

Table III. Levels of serum VEGF, BM-VEGF and BM-MVD in patients at various stages of AML.

\begin{tabular}{lccrr}
\hline & AML & AML-NR & AML-CR & AML-DFS \\
\hline B-VEGF, pg/ml & $74.97 \pm 29.04$ & $151.03 \pm 20.73$ & $146.02 \pm 40.48$ & $74.04 \pm 8.69$ \\
BM-VEGF, pg/ml & $150.58 \pm 73.36$ & $109.82 \pm 14.50$ & $109.19 \pm 49.82$ & $61.67 \pm 3.15$ \\
MVD & $22.28 \pm 6.89$ & $18.08 \pm 1.21$ & $8.89 \pm 2.60$ & $6.48 \pm 2.09$ \\
\hline
\end{tabular}

The MVD value is presented as the number of microvessels per high-power field (magnification, $\mathrm{x} 400$ ). VEGF, vascular endothelial growth factor; B-VEGF, blood plasma VEGF; BM-VEGF, bone marrow VEGF; MVD, microvessel density; AML, acute myeloid leukemia; AML-NR, no recovery from AML at 6 months post-chemotherapy; AML-CR, complete remission of AML; AML-DFS, complete recovery from AML demonstrated for 6 months post-chemotherapy.

images were captured (magnification, $x 400$ ). Individual vessels with brown-yellow stained endothelial cells were counted three times if they separated clearly from adjacent blood vessels and trabecular bone, regardless of whether there was a vascular lumen and whether the vascular lumen contained red blood cells. At least 10 staining areas of three sections from individual samples were evaluated and the value of BM-MVD was calculated for each sample.

ELISA. The concentrations of plasma and bone marrow supernatant VEGF were determined by ELISA using the VEGF ELISA kit (JingMei Biotech, Co., Ltd., Beijing, China), as previously described $(26,28,31)$. Briefly, plasma samples were diluted with sample diluents at a ratio of 1:3, while the bone marrow supernatants were diluted at a ratio of 1:5 for analysis. The samples from the various groups of patients and $\mathrm{HC}$ individuals were simultaneously analyzed, and individual samples were tested in triplicate and repeated three times. The concentrations of VEGF in individual samples were calculated, according to the standard curve established using recombinant human VEGF provided.

Data analysis. Data are expressed as the mean \pm standard deviation or the median and range if applicable. The difference between groups was analyzed by Student's $t$-test, $\chi^{2}$-test, the Mann-Whitney U test or Wilcoxon rank-sum test using SPSS 13.0 software (SPSS, Inc., Chicago, IL, USA). P $<0.05$ was considered to indicate a statistically significant difference.

\section{Results}

Significantly increased levels of serum and BM VEGF and $B M-M V D$ in patients with AML. VEGF is crucial for angiogenesis and supports the growth of solid tumors $(32,33)$. The BM-MVD is important for the pathogenesis of human leukemia (34). To examine the importance of VEGF and BM-MVD, a group of patients with different hematological disorders and 10 gender and age-matched $\mathrm{HC}$ individuals were recruited. The demographic and clinical characteristics are summarized in Table I. There is no significant difference in the distribution of age and gender. Analysis of VEGF indicated that the levels of serum VEGF in patients with AML (74.97 \pm 29.04$)$, NHL (146.89 \pm 74.67$)$ or benign anemia (122.05 \pm 45.23$)$ were significantly increased compared with the levels in the $\mathrm{HC}$ individuals $(41.76 \pm 10.03)(\mathrm{P}<0.01$; Table II). The levels of $\mathrm{BM}$ VEGF in patients with AML $(150.58 \pm 73.36)$ were significantly higher compared with the levels in the patients with NHL $(100.39 \pm 28.10)$ or benign anemia $(94.85 \pm 30.55)(\mathrm{P}<0.05)$. Furthermore, the levels of BM-MVD in patients with AML (22.28 \pm 6.89$)$ were also significantly increased compared with the levels in patients with NHL $(7.76 \pm 2.24)$ or benign anemia $(11.11 \pm 2.35)(\mathrm{P}<0.01)$. Therefore, significantly increased levels of serum and BM VEGF and BM-MVD were detected in patients with AML.

Chemotherapy alters levels of serum, BM VEGF and $B M-M V D$ in patients with AML. Following chemotherapy, 16 out of 28 patients were followed up and 9 patients (AML-CR) were completely recovered, while 7 patients (AML-NR) had not demonstrated recovery at 6 months subsequent to the last chemotherapy. The levels of serum VEGF in both AML-CR $(146.02 \pm 40.48)$ and AML-NR $(151.03 \pm 20.73)$ groups of patients were significantly increased compared with the levels prior to treatment $(74.97 \pm 29.04)(\mathrm{P}<0.01$; Table III), and there was no significant difference in the levels of 
serum VEGF between the AML-CR and AML-NR groups of patients. By contrast, the levels of BM VEGF in the AML-CR $(109.19 \pm 49.82)$ and AML-NR $(109.82 \pm 14.50)$ groups of patients were significantly lower compared with the levels prior to treatment $(150.58 \pm 73.36)(\mathrm{P}<0.05)$. Similarly, the levels of BM-MVD in the AML-CR patients were significantly lower compared with the levels present prior to treatment $(8.89 \pm 2.60$ vs. $22.28 \pm 6.89 ; \mathrm{P}<0.01)$. However, the levels of BM-MVD in the AML-NR patients were slightly reduced compared with the levels prior to treatment, but this difference was not significant $(18.08 \pm 1.21$ vs. $22.28 \pm 6.89$; $\mathrm{P}>0.05)$. Notably, the levels of BM-MVD in the AML-CR group were also significantly decreased compared with the levels in the AML-NR group $(\mathrm{P}<0.05)$. This finding indicates that a dramatic decrease in the levels of BM-MVD is associated with complete remission in patients with AML.

In addition, the levels of serum VEGF, BM VEGF and BM-MVD in 14 AML-DFS patients were also assessed. Similar levels of serum VEGF were detected in the AML and AML-DFS patients, but significantly lower levels of BM VEGF in the AML-DFS patients compared with the AML group $(61.67 \pm 3.15$ vs. $150.58 \pm 73.36$; $\mathrm{P}<0.01)$. Notably, the levels of BM-MVD in the AML-DFS group were similar to the levels in the AML-CR patients, but were significantly lower compared with the levels in the AML $(6.48 \pm 2.09$ vs. $22.28 \pm 6.89 ; \mathrm{P}<0.01)$ and AML-NR patients $(6.48 \pm 2.09$ vs. $18.08 \pm 1.21 ; \mathrm{P}<0.05)$. The present data indicate that the levels of BM-MVD vary in different stages of AML and may be inversely associated with the activity of the disease.

\section{Discussion}

VEGF is crucial for vasculogenesis and angiogenesis, and plays key roles in the development and progression of leukemia (1-3). Although angiogenesis in acute leukemia has already been extensively studied, it is unknown whether there is any difference between the Chinese population and other populations (35). In the present study, the levels of serum and BM VEGF, and BM-MVD were measured in newly diagnosed patients with AML, NHL and benign anemia. It was found that the levels of serum VEGF in patients with AML, NHL and anemia were significantly increased compared with the levels in the $\mathrm{HC}$ individuals and that the levels of serum VEGF in patients with AML were significantly decreased compared with the levels in patients with NHL or benign anemia. Furthermore, the levels of BM VEGF and BM-MVD in AML patients were significantly increased compared to patients with NHL or anemia. The present data were consistent with previous observations in other populations (36-38) and suggest that hematological disorders may promote the production of VEGF, particularly in patients with AML. The significantly increased levels of BM VEGF and BM-MVD in AML patients suggests that VEGF-associated vasculogenesis and angiogenesis may support the proliferation of malignant progenitor cells and may be associated with the pathogenesis of AML. In addition, VEGF may be a target for the design of novel therapies for AML.

Previous studies have revealed that antitumor chemotherapeutics inhibit angiogenesis and induce apoptosis of endothelial cells $(26,39)$. In the present study, it was found that subsequent to the administration of chemotherapy, significantly increased levels of serum VEGF and reduced levels of BM VEGF were detected in the patients with AML, regardless of whether they markedly responded to the therapies. Furthermore, the levels of BM-MVD in the drug-responders that completely recovered from AML were significantly reduced, compared with the levels prior to treatment. In AML-DFS patients, the levels of BM VEGF and BM-MVD were lowest compared with the levels in patients with AML prior to treatment, and in AML-NR and AML-CR patients. These data suggest that the reduced levels of BM VEGF may mitigate AML-associated vasculogenesis and angiogenesis and decrease the levels of BM-MVD in the drug-responders of AML patients. The outcome may stem from chemotherapeutic drugs that inhibit the proliferation of endothelial cells and induce endothelial cell apoptosis $(26,39)$. Provided that vasculogenesis and angiogenesis are crucial for the proliferation of malignant progenitor cells, the reduced levels of BM-MVD may contribute to complete recovery in these AML patients (40). Therefore, the measurement of BM-MVD may serve as a biomarker for evaluating the status of AML and prognosis of chemotherapeutic responses.

In summary, the present data indicated significantly increased levels of serum VEGF in patients with AML, NHL or benign anemia and that the levels of BM VEGF and BM-MVD in AML patients were significantly increased compared with patients with NHL or anemia. Treatment with chemotherapeutic agents dramatically reduced the levels of BM VEGF and BM-MVD in the AML patients, and the change in the levels of BM-MVD was associated with the drug response in AML patients. Therefore, the levels of BM-MVD may serve as a biomarker for evaluating the status of AML and the therapeutic responses in AML patients.

\section{References}

1. Ellis LM, Rosen L and Gordon MS: Overview of anti-VEGF therapy and angiogenesis. Part 1: Angiogenesis inhibition in solid tumor malignancies. Clin Adv Hematol Oncol 4 (Suppl): 1-10, 2006.

2. Hurwitz HI, Fehrenbacher L, Hainsworth JD, Heim W, Berlin J, Holmgren E, Hambleton J, Novotny WF and Kabbinavar F: Bevacizumab in combination with fluorouracil and leucovorin: An active regimen for first-line metastatic colorectal cancer. J Clin Oncol 23: 3502-3508, 2005.

3. Connolly DT: Vascular permeability factor: A unique regulator of blood vessel function. J Cell Biochem 47: 219-223, 1991.

4. Howlader N, Noone AM, Krapcho M, et al (eds): SEER Cancer Statistics Review, 1975-2009 (vintage 2009 populations), National Cancer Institute, Bethesda, MD, 2012.

5. Siegel R, Naishadham D and Jemal A: Cancer statistics, 2012. CA Cancer J Clin 62: 10-29, 2012.

6. Dores GM, Devesa SS, Curtis RE, Linet MS and Morton LM: Acute leukemia incidence and patient survival among children and adults in the United States, 2001-2007. Blood 119: 34-43, 2012.

7. Moorman AV, Chilton L, Wilkinson J, Ensor HM, Bown N and Proctor SJ: A population-based cytogenetic study of adults with acute lymphoblastic leukemia. Blood 115: 206-214, 2010.

8. PatelJP, Gönen M, Figueroa ME, Fernandez H, SunZ, Racevskis J, Van Vlierberghe P, Dolgalev I, Thomas S, Aminova O, et al: Prognostic relevance of integrated genetic profiling in acute myeloid leukemia. N Engl J Med 366: 1079-1089, 2012.

9. Ghosh K, Swaminathan S, Madkaikar M, Gupta M, Kerketta L and Vundinti B: FLT3 and NPM1 mutations in a cohort of AML patients and detection of a novel mutation in tyrosine kinase domain of FLT3 gene from Western India. Ann Hematol 91: 1703-1712, 2012. 
10. Hung CH, Yang SN, Kuo PL, Chu YT, Chang HW, Wei WJ, Huang SK and Jong YJ: Modulation of cytokine expression in human myeloid dendritic cells by environmental endocrine-disrupting chemicals involves epigenetic regulation. Environ Health Perspect 118: 67-72, 2010.

11. Todorovic M, Radisavljevic Z, Balint B, Andjelic B, Todorovic V, Jovanovic MP and Mihaljevic B: Increased angiogenesis-associated poor outcome in acute lymphoblastic leukemia: A single center study. Appl Immunohistochem Mol Morphol 20: 488-493, 2012.

12. Connolly DT, Heuvelman DM, Nelson R, Olander JV, Eppley BL, Delfino JJ, Siegel NR, Leimgruber RM and Feder J: Tumor vascular permeability factor stimulates endothelial cell growth and angiogenesis. J Clin Invest 84: 1470-1478, 1989.

13. Hahn D, Simak R, Steiner GE, Handisurya A, Susani M and Marberger M: Expression of the VEGF-receptor Flt-1 in benign, premalignant and malignant prostate tissues. J Urol 164: 506-510, 2000.

14. Fontanella C, Ongaro E, Bolzonello S, Guardascione M, Fasola G and Aprile G: Clinical advances in the development of nove VEGFR2 inhibitors. Ann Transl Med 2: 123, 2014.

15. Sahni A and Francis CW: Vascular endothelial growth factor binds to fibrinogen and fibrin and stimulates endothelial cell proliferation. Blood 96: 3772-3778, 2000

16. Keyhani A, Jendiroba DB and Freireich EJ: Angiogenesis and leukemia. Leuk Res 25: 639-645, 2001.

17. Shalaby F, Rossant J, Yamaguchi TP, Gertsenstein M, Wu XF, Breitman ML and Schuh AC: Failure of blood-island formation and vasculogenesis in Flk-1-deficient mice. Nature 376: 62-66, 1995.

18. Song GL and Han ZC: Malignant hematologic disease and angiogenesis. Zhonghua Xue Ye Xue Za Zhi 23: 217-219, 2002 (In Chinese).

19. Kampen KR, Ter Elst A and de Bont ES: Vascular endothelial growth factor signaling in acute myeloid leukemia. Cell Mol Life Sci 70: 1307-1317, 2013

20. Kvasnicka HM and Thiele J: Bone marrow angiogenesis: Methods of quantification and changes evolving in chronic myeloproliferative disorders. Histol Histopathol 19: 1245-1260, 2004.

21. Kuzu I, Beksac M, Arat M, Celebi H, Elhan AH and Erekul S: Bone marrow microvessel density (MVD) in adult acute myeloid leukemia (AML): Therapy induced changes and effects on survival. Leuk Lymphoma 45: 1185-1190, 2004.

22. Du W, Hattori Y, Hashiguchi A, Kondoh K, Hozumi N, Ikeda Y, Sakamoto M, Hata J and Yamada T: Tumor angiogenesis in the bone marrow of multiple myeloma patients and its alteration by thalidomide treatment. Pathol Int 54: 285-294, 2004

23. Perez-Atayde AR, Sallan SE, Tedrow U, Connors S, Allred E and Folkman J: Spectrum of tumor angiogenesis in the bone marrow of children with acute lymphoblastic leukemia. Am J Pathol 150: 815-821, 1997.

24. Padró T, Ruiz S, Bieker R, Bürger H, Steins M, Kienast J, Büchner T, Berdel WE and Mesters RM: Increased angiogenesis in the bone marrow of patients with acute myeloid leukemia. Blood 95 $2637-2644,2000$

25. Harris NL, Jaffe ES, Diebold J, Flandrin G, et al: World Health Organization classification of neoplastic diseases of the hematopoietic and lymphoid tissues: Report of the Clinical Advisory Committee meeting-Airlie House, Virginia, November 1997. J Clin Oncol 17: 3835-3849, 1999.
26. Mangi $\mathrm{MH}$ and Newland AC: Angiogenesis and angiogenic mediators in hematological malignancies. Br J Hematol 111: 43-51, 2000.

27. Swerdlow SH, Campo E, Harris NL, et al (eds): WHO Classification of Tumors of Haematopoietic and Lymphoid Tissues. 4th Edition. IARC Press, Lyon, France, 2008.

28. Kini AR, Peterson LA, Tallman MS and Lingen MW Angiogenesis in acute promyelocytic leukemia: Induction by vascular endothelial growth factor and inhibition by all-trans retinoic acid. Blood 97: 3919-3924, 2001.

29. Vacca A, Ribatti D, Roncali L, Ranieri G, Serio G, Silvestris F and Dammacco F: Bone marrow angiogenesis andprogression in multiple myeloma. Br J Haematol 87: 503-508, 1994.

30. Weidner N: Current pathologic methods for measuring intratumoral microvessel density within breast carcinoma and other solid tumors. Breast Cancer Res Treat 36: 169-180, 1995.

31. List AF, Glinsmann-Gibson B, Stadheim C, Meuillet EJ, Bellamy W and Powis G: Vascular endothelial growth factor receptor-1 and receptor-2 initiate a phosphatidylinositide 3-kinase-dependent clonogenic response in acute myeloid leukemia cells. Exp Hematol 32: 526-535, 2004.

32. Kuo CJ, Farnebo F, Yu EY, Christofferson R, Swearingen RA, Carter R, von Recum HA, Yuan J, Kamihara J, Flynn E, et al: Comparative evalution of the antitumor activity of antiangiogenic proteins delivered by gene transfer. Proc Natl Acad Sci USA 98: 4605-4610, 2001.

33. Kendall RL, Wang G and Thomas KA: Identification of a natural soluble form of the vascular endothelial growth factor receptor, FLT-1 and its heterodimerization with KDR. Biochem Biophys Res Commun 226: 324-328, 1996.

34. Lin P, Sankar S, Shan S, Dewhirst MW, Polverini PJ, Quinn TQ and Peters KG: Inhibition of tumor growth by targeting tumor endothelium using a soluble vascular endothelial growth factor receptor. Cell Growth Differ 9: 49-58, 1998.

35. Haouas H: Angiogenesis and acute myeloid leukemia. Hematology 19: 311-323, 2014.

36. de Bont ES, Fidler V, Meeuwsen T, Scherpen F, Hählen K and Kamps WA: Vascular endothelial growth factor secretion is an independent prognostic factor for relapse-free survival in pediatric acute myeloid leukemia patients. Clin Cancer Res 8: 2856-2861, 2002.

37. Dias S, Hattori K, Heissig B, Zhu Z, Wu Y, Witte L, Hicklin DJ, Tateno M, Bohlen P, et al: Inhibition of both paracrine and autocrine VEGF/VEGFR-2 signaling pathways is essential to induce long-term remission of xenotransplanted human leukemias. Proc Natl Acad Sci USA 98: 10857-10862, 2001.

38. Rodriguez-Ariza A, Lopez-Pedrera C, Aranda E and Barbarroja N: VEGF targeted therapy in acute myeloid leukemia. Crit Rev Oncol Hematol 80: 241-256, 2011.

39. Zhang ZN (ed): Diagnosis and treatment criteria of hematopathy. 2nd edition. Beijing: Science Press, Beijing, 1998.

40. Song G, Li Y and Jiang G: Role of VEGF/VEGFR in the pathogenesis of leukemias and as treatment targets (Review). Oncol Rep 28: 1935-1944, 2012. 